Document downloaded from:

http://hdl.handle.net/10251/93518

This paper must be cited as:

J.D. Badia; Strömberg, E.; Karlsson, S.; A. Ribes-Greus (2012). The role of crystalline, mobile amorphous and rigid amorphous fractions in the performance of recycled poly (ethylene terephthalate) (PET). Polymer Degradation and Stability. 97(1):98-107. doi:10.1016/j.polymdegradstab.2011.10.008

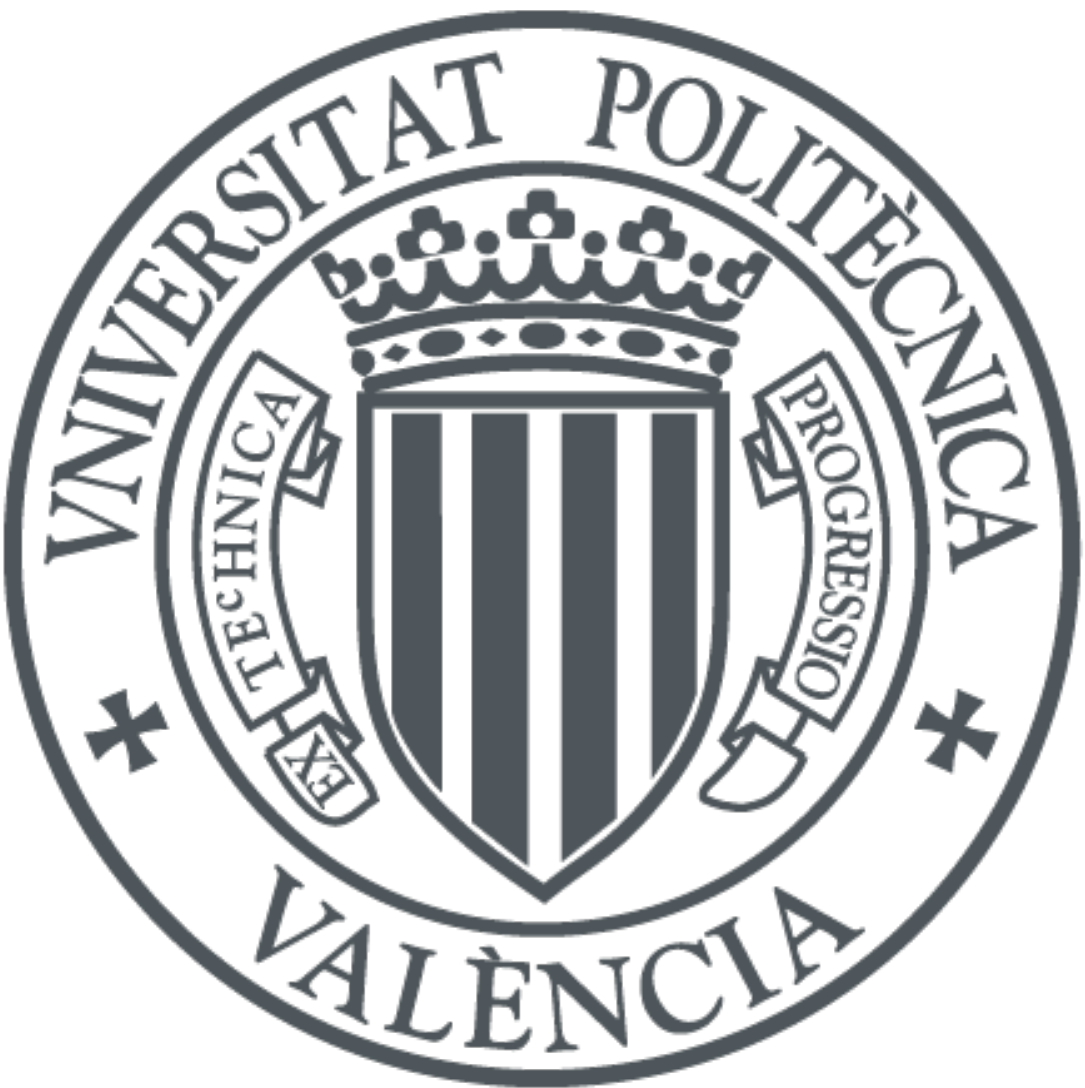

The final publication is available at

http://doi.org/10.1016/j.polymdegradstab.2011.10.008

Copyright ELSEVIER SCI LTD

Additional Information 
J.D. Badia, E. Strömberg, S. Karlsson, A. Ribes-Greus, The role of crystalline, mobile amorphous and rigid amorphous fractions in the performance of recycled poly (ethylene terephthalate) (PET). Polymer Degradation and Stability,97 (1) 98-107, 2012

THE ROLE OF CRYSTALLINE, MOBILE AMORPHOUS AND RIGID AMORPHOUS FRACTIONS ON THE PERFORMANCE OF RECYCLED POLY (ETHYLENE TEREPHTHALATE) (PET)

\author{
J.D. Badia' ${ }^{1}$ E. Strömberg², S. Karlsson ${ }^{2,3}$, A. Ribes-Greus ${ }^{1, *}$
}

This is an open-access version, according to http://www.sherpa.ac.uk/romeo/issn/0141-3910/

Full text available at http://www.sciencedirect.com/science/article/pii/S0141391011003351

DOI: 10.1016/j.polymdegradstab.2011.10.008

Please, cite it as:

J.D. Badia, E. Strömberg, S. Karlsson, A. Ribes-Greus, The role of crystalline, mobile amorphous and rigid amorphous fractions in the performance of recycled poly (ethylene terephthalate) (PET). Polymer Degradation and Stability,97 (1) 98-107, 2012

\footnotetext{
${ }^{1}$ Instituto de Tecnología de Materiales (ITM), Universitat Politècnica de València

Camino de Vera s/n, E-46022 Valencia, Spain

${ }^{2}$ School of Chemical Science and Engineering,
} Fibre and Polymer Technology, KTH - Royal Institute of Technology, Teknikringen 56-58, SE-10044 Stockholm, Sweden ${ }^{3}$ Presently, Vice Chancellor Skövde University, SE-541 28 SKÖVDE, Sweden *corresponding author: A. Ribes-Greus aribes@ter.upv.es 
J.D. Badia, E. Strömberg, S. Karlsson, A. Ribes-Greus, The role of crystalline, mobile amorphous and rigid amorphous fractions in the performance of recycled poly (ethylene terephthalate) (PET). Polymer Degradation and Stability,97 (1) 98-107, 2012

\title{
THE ROLE OF CRYSTALLINE, MOBILE AMORPHOUS AND RIGID AMORPHOUS FRACTIONS ON THE PERFORMANCE OF RECYCLED POLY (ETHYLENE TEREPHTHALATE) (PET)
}

\author{
J.D. Badia, E. Strömberg, S. Karlsson, A. Ribes-Greus
}

\section{Keywords}

poly(ethylene terephthalate) (PET), mechanical recycling, differential scanning calorimetry (DSC), infrared analysis (FT-IR), mechanical properties

\begin{abstract}
The action of thermo-mechanical degradation induced by mechanical recycling on poly (ethylene terephthalate) was simulated by successive injection moulding cycles. Degradation reactions provoked chain scissions and a reduction in molar mass mainly driven by the reduction of diethyleneglycol to ethylene glycol units in the flexible domain of the PET backbone, and the formation of -OH terminated species with shorter chain length. The consequent microstructural changes were quantified taking into account a threefraction model involving crystalline, mobile amorphous (MAF) and rigid amorphous fractions (RAF). A remarkable increase of RAF, to a detriment of MAF was observed, while the percentage of crystalline fraction remained nearly constant. A deeper analysis of the melting behaviour, the segmental dynamics around the glass-rubber relaxation, and the macroscopic mechanical performance, showed the role of each fraction leading to a loss of thermal, viscoelastic and mechanical features, particularly remarkable after the first processing cycle.
\end{abstract}


J.D. Badia, E. Strömberg, S. Karlsson, A. Ribes-Greus, The role of crystalline, mobile amorphous and rigid amorphous fractions in the performance of recycled poly (ethylene terephthalate) (PET). Polymer Degradation and Stability,97 (1) 98-107, 2012

\section{Introduction}

The extended use of poly(ethylene terephthalate) (PET) over the last decades, mainly in the food packaging sector, is due to its excellent mechanical, chemical, processing and thermal properties. Consequently, the amount of post-consumer PET present in urban solid waste is high and has to be managed. Among all recovery methods, mechanical recycling represents one of the most successful processes and has received considerable attention due to its main advantages. During its life cycle (synthesis, processing, use, discarding, cleaning, recycling), PET is subjected to the interaction of degradation agents such as oxygen, light, mechanical stresses, temperature or water. Individually or synergically, these agents may provoke the breakage of the macromolecules and diminish the processability and final performance [1-3]. In this sense, the application of protocols to simulate the degradation subjected by polymers during recycling has been effectively performed for commodities such as polyethylene (PE) [4], polypropylene (PP) [4], polystyrene (PS) [5-6], poly (vinyl chloride) (PVC) [7] or PET [8-14].

Reported analyses for PET generally showed a loss of mechanical [10-11, 14-15] thermal [8, 14-15] or rheological [11, 15] properties. Further information can be found in the review by Awaja and Pavel [16]. The majority of studies explained the effects of reprocessing taking into account a two-fraction model, in which only amorphous and crystalline domains were present, and thus related the changes in properties to the relative balance between both fractions [10]. Nevertheless, different studies performed by means of techniques such as X-ray scattering techniques [17-19], Fourier-Transform Infra-Red Analysis (FTIR) [20], Temperature-Modulated Differential Scanning Calorimetry (TMDSC) [21-22] , Thermo-Mechanical Analysis (TMA) [23] or micro-indentation [19] showed the suitability of a three-fraction model to describe the microstructure of PET. With this model, mobile (composed by the mobile amorphous fraction MAF) and rigid (formed by a crystalline $C$ and a rigid amorphous fraction $R A F$ ) domains are defined [24-25]. The $R A F$ is normally assigned to both the amorphous-crystalline interface in basal lamellar planes [26-28], in connection to the bulk amorphous fraction $M A F$, as well as to the inter-spherulitic domains constrained between crystalline lamellae stacks [25]. On the other hand, the $M A F$, which normally behaves contributing to the heat capacity jump throughout the glass transition, is associated to the freely interstack regions [29].

In this context, the aim of this work was firstly focused on testing the structural groups affected by chain scission induced by thermo-mechanical degradation. Afterwards, the evolution of the microstructure throughout the reprocessing stages was assessed by thermal analysis, according to a three-fraction model to understand the effects of thermo-mechanical degradation on PET. Consequently, the performance of reprocessed PET was correlated with the crystalline, mobile amorphous and rigid amorphous fractions. The methodology applied allowed to establish an accurate control of the quality of recycled PET for its suitable material valorisation. 
J.D. Badia, E. Strömberg, S. Karlsson, A. Ribes-Greus, The role of crystalline, mobile amorphous and rigid amorphous fractions in the performance of recycled poly (ethylene terephthalate) (PET). Polymer Degradation and Stability,97 (1) 98-107, 2012

\section{Experimental procedure and calculations}

\subsection{Material, reprocessing simulation and sample preparation}

Poly (ethylene terephthalate) (PET) SEDAPET SP04 was a bottle-grade PET obtained from Catalana de Polimers S.A., Grup LaSeda (Barcelona, Spain) in the form of pellets. Prior to processing, virgin PET (VPET) pellets were dried during $5 \mathrm{~h}$ at $160{ }^{\circ} \mathrm{C}$ in a dehumidifier Conair Micro-D FCO 1500/3 (UK), in order to remove as much humidity as possible from the PET flakes. Afterwards, the samples were processed by means of injection moulding employing an Arburg 420 C 1000-350 (Germany) injector, single-screw model (diameter $\Phi=35 \mathrm{~mm}$, length $\Phi=23$ ). Successive processing steps were applied under the same conditions, being the samples carefully dried before each processing cycle. Temperature gradient set from hopper to die was $270,275,280,285$ and $280{ }^{\circ} \mathrm{C}$. Moulds were set at $15^{\circ} \mathrm{C}$. Cooling time residence was 40 $\mathrm{s}$ and total residence time nearly $60 \mathrm{~s}$. After injection, a fraction of the samples was kept as test specimen and the rest was ground by means of a cutting mill Retsch SM2000 (UK), which provided pellets of size $\Phi$ $<20 \mathrm{~mm}$ to be fed back into the recirculation process. Up to five processing cycles were applied to obtain the different testing specimens of reprocessed PET (RPET-i, with i: 1-5).

Prismatic probes of $1 \mathrm{~mm}$ of thickness were prepared for DMTA characterization by melt compression in a Collin PCS-GA Type Press 800 (GA, USA) preset at $260{ }^{\circ} \mathrm{C}$. Five pressure steps were performed with the program: 5 minutes at 4 bar, 3 minutes at 100 bar, 1 minutes at 80 bar, 5 minutes at 180 bar, and 15 minutes at 75 bar.

\subsection{Mechanical performance}

Tensile testing was carried out at laboratory conditions 23/50, according to ISO 291, atmosphere 23/50, class 1 [30]. Tensile tests were performed on reprocessed PET in order to investigate the changes in macroscopic mechanical properties, on dumbbell samples following ISO 527-2 (type 1A) [31], by means of an Instron 5566 universal electromechanical testing instrument (Instron Corp, MA, USA), at a crosshead speed of $10 \mathrm{~mm} \cdot \mathrm{min}^{-1}$, a $10 \mathrm{kN}$ load cell and gauche length of $50 \mathrm{~mm}$. Analyses were repeated at least 6 times per material, and the average of elastic modulus, elongation at break and stress at break were used as representative values.

Charpy impact experiments were carried out following ISO 179 [32], with a hammer of $1 \mathrm{~J}$ and a notch size radius of $1,5 \mathrm{~mm}$, under the same laboratory conditions than tensile tests. Analyses were repeated at least 5 times per material, and the averages were taken as representative values.

\subsection{Scanning Electron Microscopy (SEM)}

The morphology of the specimens was analysed by means of a Hitachi S-4800 Field Emission Scanning Electron Microscope (Tokyo, Japan). The samples from each material were prepared by cutting squared pieces from a randomly chosen part of the processed specimen. The pieces were mounted on metal studs and sputter-coated with a $2 \mathrm{~nm}$ gold layer using a Cressington 208HR high resolution sputter coater (Watford, UK), equipped with a Cressington thickness monitor controller. 
J.D. Badia, E. Strömberg, S. Karlsson, A. Ribes-Greus, The role of crystalline, mobile amorphous and rigid amorphous fractions in the performance of recycled poly (ethylene terephthalate) (PET). Polymer Degradation and Stability,97 (1) 98-107, 2012

\subsection{Fourier-Transform Infrared (FT-IR) Analysis}

FT-IR spectra were collected by a NEXUS Thermo Nicolet 5700 FT-IR Spectrometer (MA, USA), previously calibrated, and equipped with a single-reflection Smart Performer accessory for attenuated total reflection (ATR) measurements, with diamond crystal. 32 co-added spectra were recorded for each specimen at a resolution of $4 \mathrm{~cm}^{-1}$ with a spacing of $1 \mathrm{~cm}^{-1}$, from 4000 to $600 \mathrm{~cm}^{-1}$ of wavenumber. IR spectra were analyzed with the help of OMNIC 7.0 software from Thermo Scientific. The peak at $1410 \mathrm{~cm}^{-}$

${ }^{1}$ was used for normalization [33] before any data processing, corresponding to the benzene ring in-plane deformation, which is usually used as internal standard due to it is not sensitive to effects of orientation or conformation [34], and it is useful to correct possible variations arisen from defects in surface quality or sample positioning. At least 8 measurements per material were performed at different locations of the plate, in order to obtain representative results. Presented spectra correspond to the average of each individual analysis.

\subsection{Molar mass determination}

The intrinsic viscosity [ $\eta$ ] was measured according to the standard ISO 1628-1 [35], by means of a CannonFenske capillary viscosimeter type at $25^{\circ} \mathrm{C}$, with the use of $60 / 40 \mathrm{wt} \%$ phenol/1,1,2,2-tetracholoethane as solvent. Dissolutions of pellets ranged from 0.1 to $0.5 \mathrm{~g} \cdot \mathrm{dL}^{-1}$. Measurements were performed by quintuplicate for each concentration $c$ and $[\eta]$ was obtained from extrapolation to $c \rightarrow 0$ of Huggins and Kraemer plots [36], which respectively account for the variation of reduced $\eta_{\text {red }}$ and inherent $\eta_{\text {inh }}$ viscosities with the concentration, being $\eta_{r e d}=c^{-1} \cdot \eta_{s p}, \eta_{\text {inh }}=c^{-1} \cdot \ln \eta_{r e l}, \eta_{s p}=\eta_{r e l}-1$ and $\eta_{r e l}=t \cdot t_{0}^{-1}$, where $t$ and $t_{0}$ were the times (s) of flowing of the dissolution and solvent, respectively. Fakirov [37] and Jabarin [38] proposed the equations $[\eta]=K_{n} \cdot M_{n}{ }^{\alpha n}$ and $[\eta]=K_{w} \cdot M_{w}{ }^{\alpha w}$ to calculate the number and weight average molar mass values $\left(M_{n}, M_{w} \mathrm{~g} \cdot \mathrm{mol}^{-1}\right)$. The constants $K_{n}=3.72 \cdot 10^{-4} \mathrm{dL} \cdot \mathrm{g}^{-1}$ and $\alpha n=0.73$, and $K_{w}=4.68 \cdot 10^{-4} \mathrm{dL} \cdot \mathrm{g}^{-1}$ and $\alpha w=0.68$ were used. The polydispersity index was calculated as $P D I=M_{w} \cdot M_{n}{ }^{-1}$. It has been shown [39] that these parameters are suitable for monitoring degradation processes.

\subsection{Differential Scanning Calorimetry (DSC)}

DSC analyses were carried out by a Mettler Toledo DSC 820 instrument (Columbus, OH) calibrated with indium and zinc standards. Approximately $5 \mathrm{mg}$ of pellets were placed in $40 \mu \mathrm{L}$ aluminium pans, which were sealed and pierced to allow the $\mathrm{N}_{2}$ gas flow $\left(50 \mathrm{~mL} \cdot \mathrm{min}^{-1}\right)$. A heating/cooling/heating program with a $+/-2^{\circ} \mathrm{C} \cdot \mathrm{min}^{-1}$ rate was employed in the temperature range between $25^{\circ} \mathrm{C}$ and $290{ }^{\circ} \mathrm{C}$. The samples were characterized with the aid of the software $\operatorname{STAR}^{\mathrm{e}}$ 9.10, at least by triplicate, and the averages were taken as representative values.

The glass transition was characterized according to ISO 11357-2 [40] and the temperature in its inflection point $\left(T_{g}\right)$ and the jump in heat capacity $\left(\Delta C_{p}\right)$ were recorded. Crystallization and melting phenomena were assessed in terms of their onset and peak temperatures, along with the enthalpies related to their areas to common baselines.

According to a three-fraction model, the relative values of the crystalline $\left(X_{C}\right)$, mobile amorphous fraction $\left(X_{M A F}\right)$ and rigid amorphous fraction $\left(X_{R A F}\right)$, were calculated as follows: 


$$
\begin{gathered}
X_{C}=\frac{\Delta h_{m}-\sum \Delta h_{C C}}{\Delta h_{m}^{0}} \\
X_{M A F}=\frac{\Delta C_{p}}{\Delta C_{p}^{0}} \\
X_{R A F}=1-X_{M A F}-X_{C}
\end{gathered}
$$

, being $\Delta h_{m}$ and $\Delta h_{C}$ the specific melting and cold-crystallization enthalpies $\left(\mathrm{J} \cdot \mathrm{g}^{-1}\right), \Delta h_{m}{ }^{0}$ the melting enthalpy of a fully crystalline PET (140 J.g $\left.{ }^{-1}[41]\right)$, and $\Delta C_{p}^{0}$ the heat capacity increment of a fully amorphous PET $\left(0.405 \mathrm{~J} \cdot \mathrm{g}^{-1} \cdot \mathrm{K}^{-1}[42]\right)$.

\subsection{Dynamical-Mechanical Thermal Analysis (DMTA)}

DMTA test were conducted in dual cantilever clamping with $10 \mathrm{~mm}$ of effective length between clamps, in bending mode, by means of a DMA/SDTA861 ${ }^{\mathrm{e}}$ Dynamic Mechanical Analyzer, from Mettler-Toledo (OH, USA). Experiments were carried out from $25^{\circ} \mathrm{C}$ to $175^{\circ} \mathrm{C}$ with isothermal steps of $2^{\circ} \mathrm{C}$, measuring 8 frequencies per decade between 0.1 and $100 \mathrm{~Hz}$. 
J.D. Badia, E. Strömberg, S. Karlsson, A. Ribes-Greus, The role of crystalline, mobile amorphous and rigid amorphous fractions in the performance of recycled poly (ethylene terephthalate) (PET). Polymer Degradation and Stability,97 (1) 98-107, 2012

\section{Results and discussion}

\subsection{Structural changes induced by thermo-mechanical degradation}

The absorbance spectra of virgin poly (ethylene terephthalate) (VPET) and its successive recyclates (RPETi, i: 1-5) were recorded by Fourier-Transform Infrared Spectroscopy (FT-IR). Typical PET spectra were obtained for all samples. Assignments of IR bands can be found elsewhere [43-44]. Interesting structural changes could be deduced by inspection of Figure 1 and Figure 2, which respectively show the evolution of the absorption of the stretching vibration bands of the ethylene group associated to the glycol units of PET, and the development of a polymeric-bonded -OH end-group. Different studies [45-46] reported the FTIR analysis of combinations of PET with poly(ethylene glycol) (PEG). The bands situated near $2962 \mathrm{~cm}^{-}$ ${ }^{1}$ and $2903 \mathrm{~cm}^{-1}$ correspond to the asymmetrical $v_{a s}\left(\mathrm{CH}_{2}\right)$ and symmetrical $v_{s}\left(\mathrm{CH}_{2}\right)$ stretching vibrations of the ethylene glycol (EG) domains of PET. These bands shift to around $2918 \mathrm{~cm}^{-1}$ and $2854 \mathrm{~cm}^{-1}$ in case diethylene glycol domains (DEG) are present in PET backbone. Figure 1 shows that both VPET and RPET1 absorbed at $2918 \mathrm{~cm}^{-1}$ and $2854 \mathrm{~cm}^{-1}$. However, these bands vanished for the rest of recyclates. This effect is in agreement with the mechanisms described in a previous study [9] in which the degradation of VPET was explained by the loss of EG units via mainly intramolecular transesterifications or homolytic reactions, such as chain scission by hydrolysis. On the other hand, the increase of the band centred around $3548 \mathrm{~cm}^{-}$ ${ }^{1}$ (Figure 2) suggested the promotion of -OH terminated species with more reprocessing cycles, also in agreement with our previous results [9]. The increase of these species may explain the increased yellowed appearance of PET recyclates with each processing step, due to they can act as precursors of discoloration reactions, as shown by Choudhury et al. [47]. This feature impoverishes RPET possibilities to be employed in food-packaging goods.
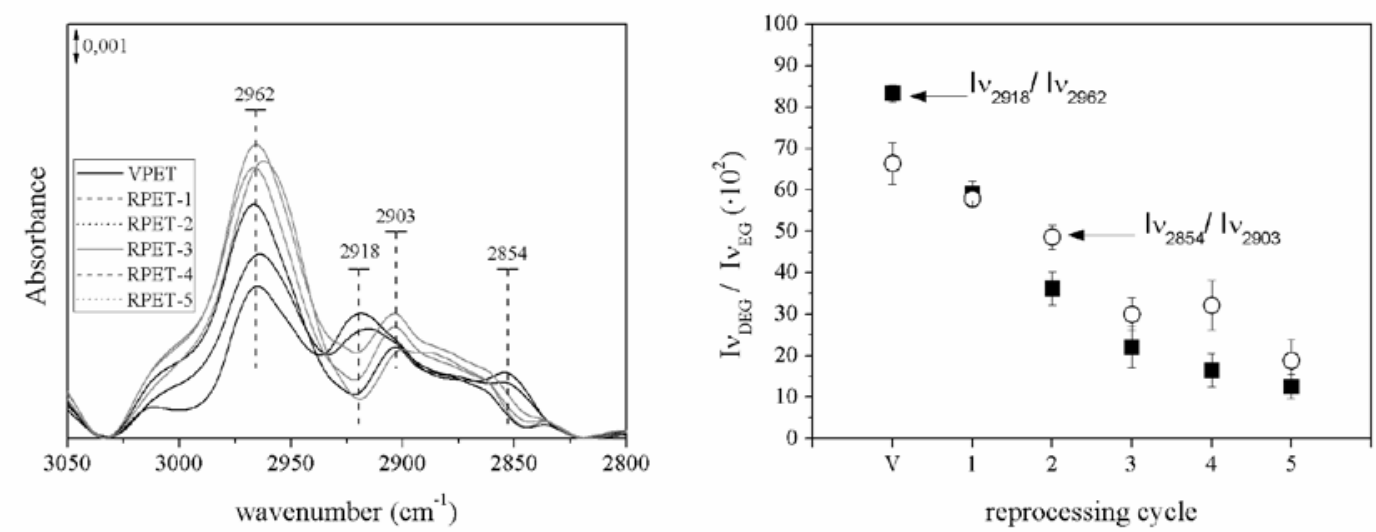

Figure 1. FT-IR analysis of the C-H stretching region: (left) FT-IR spectra evolution; (right) semiquantitative analysis. 

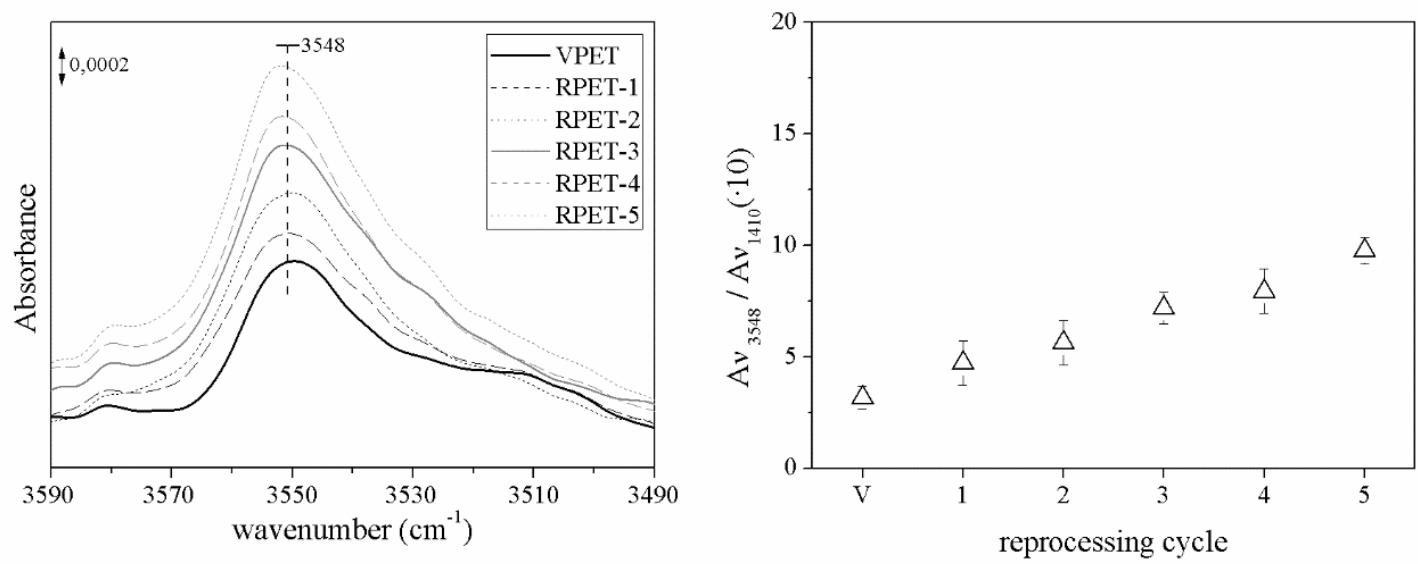

Figure 2. O-H stretching vibration study: (left) FT-IR spectra evolution; (right) semiquantitave analysis.

The nature of the $-\mathrm{OH}$ terminated species, mainly hydroxyl and carboxyl species, to act as auto-catalysers during thermo-mechanical degradation may be an evidence of reduction of molar mass. Thus, the evolution of the molar masses was correlated to the number of recyclates, as shown in Figure 3. The linear correlation values $\left(R^{2}\right)$ obtained for both Huggins and Kraemer plots were higher than 0.925 for all materials. An exponential profile for the overall molar mass decay was found, where two different stages were distinguished. Both $M_{w}$ and $M_{n}$ decreased steeply with each reprocessing step during the two first processing cycles, with marginal drops of $35 \%$ from VPET to RPET-1 and 32 \% from RPET-1 to RPET-2 in the case of $M_{w}$. From the second recyclate on, the tendency was attenuated and the variations showed an asymptotic profile towards slightly inferior values for further recyclates. Consequently, the polydispersity index showed a similar value after the second recyclate, related to likely similar distributions of chains.

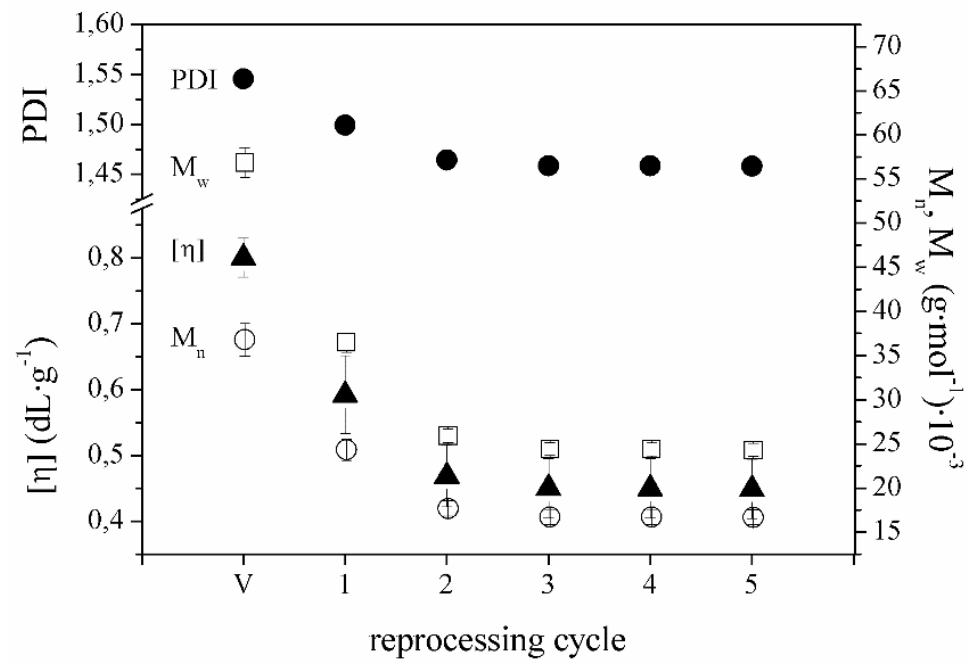

Figure 3. Evolution of intrinsic viscosity, molar masses and polydispersity index. 
The significant decrease in molar mass may affect the processability and homogeneity of PET recyclates. Surface characterization of reprocessed PET by SEM shown at Figure 4 exhibited typical morphologies of industrially-processed polymers. The micrographs of RPET-5 presented a loss of the orientation of the processing lines shown by VPET. In addition, the surface seemed to be less smooth and more liable to mechanical scratches. This effect has been found for other recycled commodities such as PE, PP or PS [4, 6]. As well, inefficiently melted pellets were adhered to the surface, thus indicating a loss of processability of RPET-5 under the same experimental conditions of VPET. The morphological defects may arise as a result of new structural and conformational arrangements between the amorphous and crystalline regions of PET and thus a deep characterization is given afterwards.
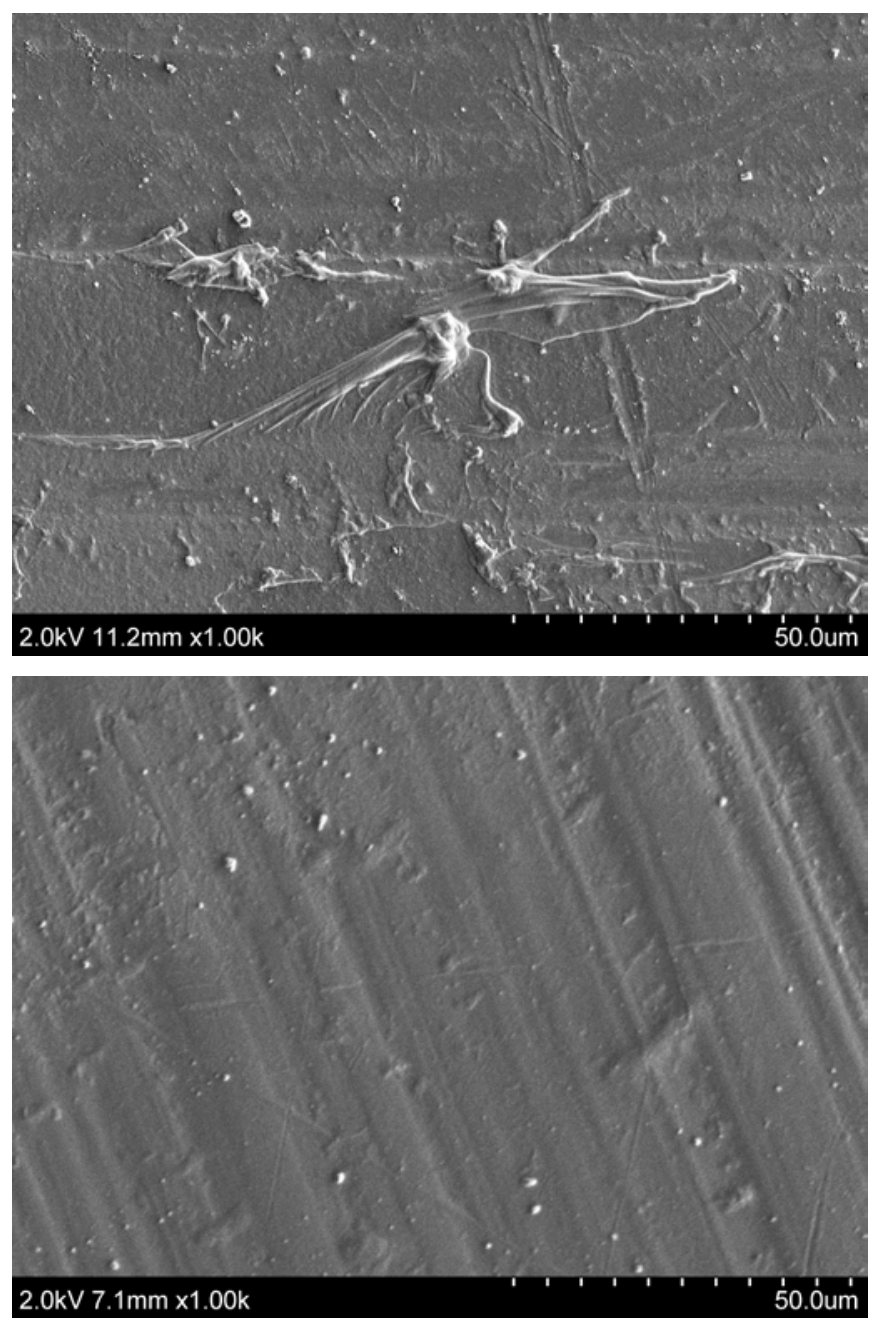

Figure 4. Surface analysis by SEM. (a: VPET, b: RPET-5)

\subsection{Influence of reprocessing on the microstructure of PET}

Differential Scanning Calorimetric (DSC) and FT-IR analysis were applied to assess the microstructural changes induced by successive reprocessing to PET. Figure 5 shows the results of the use of the threefraction model to explain the evolution of the ratios of crystalline $\left(X_{C}\right)$, mobile amorphous $\left(X_{M A F}\right)$ and rigid amorphous $\left(X_{R A F}\right)$ fractions. An initial $\left(X_{C}, X_{M A F}, X_{R A F}\right)$ distribution of $~(29 / 53 / 18) \%$ was found for VPET, similar to that reported in other studies [48]. This distribution changed to $\sim(27 / 44 / 29) \%$ after the second 
injection and to $\sim(32 / 36 / 35) \%$ after the fifth injection. The major change happened from the first to the second recyclate, involving a marginal modification close to a $10 \%$ for both $X_{M A F}$ and $X_{R A F}$. The results suggested that the $M A F$ was the principally attacked zone by thermo-mechanical degradation, releasing shorter chains that might fold among themselves and rearrange into inter-crystalline domains, increasing the $R A F$ with each reprocessing cycle.

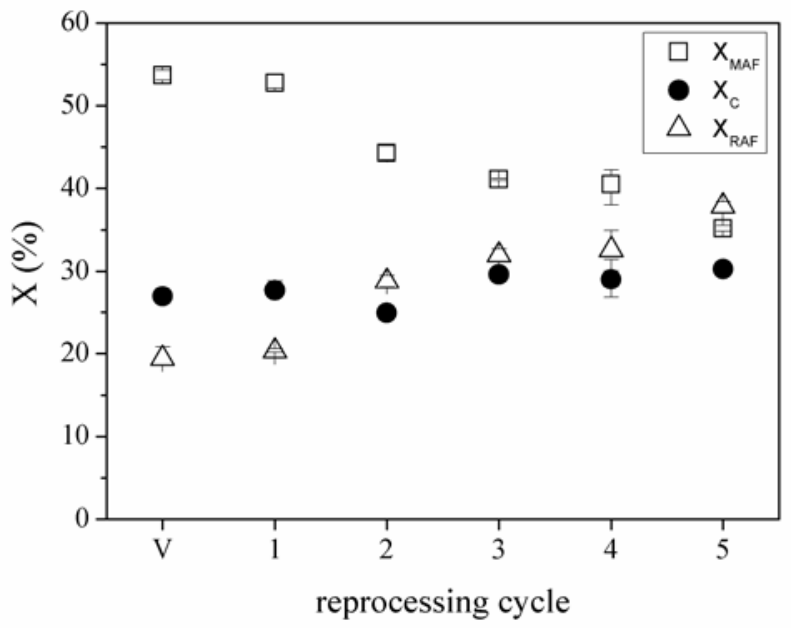

Figure 5. Evolution of the balance between crystalline and mobile and rigid amorphous fractions

The FT-IR analysis of specific gauche/trans pairs was also useful to characterize the changes in morphology of PET. The vibrations of the ethylene group of the glycol unit of PET exist in two forms, a gauche or relaxed form, present in the amorphous phase of PET; and a trans, or extended form, which is more present in the crystalline fraction of PET. The bands at $1471 \mathrm{~cm}^{-1}$ and $1466 \mathrm{~cm}^{-1}$ form the gauche/trans pair of the C-H bending vibration of the glycol unit in PET, whereas the bands at $899 \mathrm{~cm}^{-1}$ and $846 \mathrm{~cm}^{-1}$ form the respective for the rocking vibration of the same group. Figure 6 shows a decrease of the relative ratio of the absorbance areas for both vibration bands. As the increment of the crystalline fraction was not significantly prominent, it did not justify this decrease. Therefore, the decrease in the gauche/trans ratio indicated a reduction of the amorphous domains due to reprocessing. This change was especially remarkable after the second recyclate, fact that may be related to the reduction from diethylene glycol to ethylene glycol in the PET backbone, as explained above. After reprocessing, the new structures with shorter backbones and less flexible glycol units may rearrange into more packed structures. 


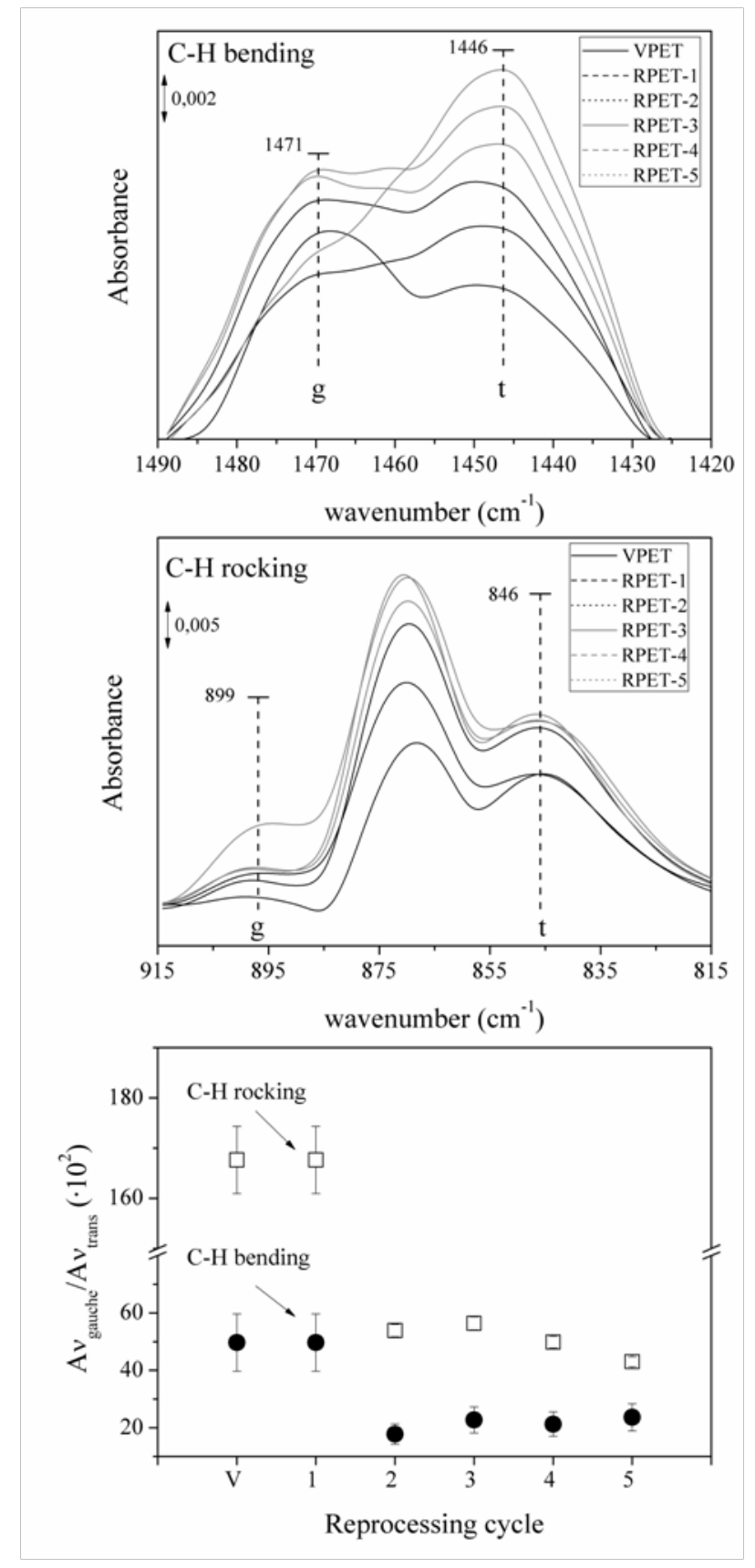

Figure 6. C-H vibrations study: (upper) FT-IR spectra evolution of C-H bending band; (middle)

FT-IR spectra evolution of C-H rocking band; (lower) semiquantitave analysis.

\subsection{An extended study of the crystalline fraction}

Further analysis evidenced that the morphological changes underwent by PET facing successive injectionmoulding operations gave rise to different crystalline domains, as shown in previous reports [8]. The influence of reprocessing on the crystallization behaviour was studied from the cooling thermograms (Figure 7); considering onset $\left(T_{C 0}\right)$ and peak crystallization temperatures $\left(T_{C}\right)$, crystallization temperature range $\left(\Delta T_{C}\right)$ and specific crystallization enthalpy $\left(\Delta h_{C}\right)$ for the assessment, which values are gathered at 
Table 1. The low cooling rate used in the DSC analysis promoted the formation of nuclei for the crystallization, and it was therefore useful for the comparison between the formation of crystalline domains of virgin and reprocessed PET. The cooling thermograms appeared earlier, sharper and steeper at the beginning of the crystallization, as well as involving bigger enthalpies with each reprocessing cycle, being particularly remarkable the change from RPET-1 to RPET-2. The $T_{C}$ was appointed as a quality parameter to distinguish between virgin and reprocessed PET in previous reports [8, 49], and therefore its reduction pointed out the presence of shorter chains with each reprocessing cycle. The whole crystalline fraction formed during cooling was melted during subsequent DSC heating, as shown in Table 1 by the values of specific melting enthalpy $\Delta h_{m}$ similar to those of crystallization $\Delta h_{C}$.

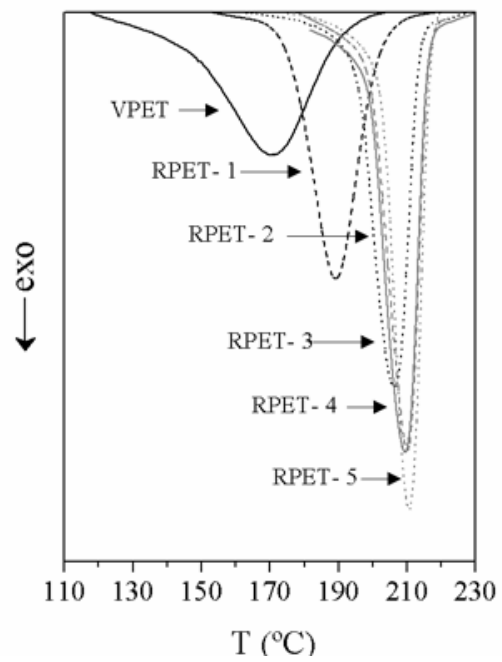

Figures 7. Evolution of DSC cooling thermograms

Table 1. Calorimetric results

\begin{tabular}{|l|l|l|l|l|l|l|}
\hline Material & $\mathrm{T}_{\mathrm{g}}\left({ }^{\circ} \mathrm{C}\right)$ & $\mathrm{T}_{\mathrm{C} 0}\left({ }^{\circ} \mathrm{C}\right)$ & $\Delta \mathrm{T}_{\mathrm{C}}\left({ }^{\circ} \mathrm{C}\right)$ & $\mathrm{T}_{\mathrm{C}}\left({ }^{\circ} \mathrm{C}\right)$ & $\Delta \mathrm{h}_{\mathrm{C}}\left(\mathrm{J} \cdot \mathrm{g}^{-1}\right)$ & $\Delta \mathrm{h}_{\mathrm{m}}\left(\mathrm{J} \cdot \mathrm{g}^{-1}\right)$ \\
\hline VPET & $77.6 \pm 0.5$ & $146.3 \pm 0.5$ & $54.2 \pm 0.4$ & $171.6 \pm 2.6$ & $-35.8 \pm 2.1$ & $37.7 \pm 1.1$ \\
\hline RPET-1 & $77.1 \pm 0.3$ & $165.5 \pm 0.5$ & $37.1 \pm 0.8$ & $189.0 \pm 0.6$ & $-36.4 \pm 0.2$ & $38.7 \pm 1.3$ \\
\hline RPET-2 & $76.4 \pm 0.1$ & $185.3 \pm 2.3$ & $32.2 \pm 1.7$ & $204.7 \pm 3.1$ & $-38.1 \pm 1.4$ & $35.2 \pm 0.4$ \\
\hline RPET-3 & $76.2 \pm 0.1$ & $189.2 \pm 0.8$ & $29.1 \pm 1.1$ & $208.3 \pm 1.3$ & $-40.2 \pm 0.9$ & $41.4 \pm 0.5$ \\
\hline RPET-4 & $76.3 \pm 0.7$ & $188.1 \pm 0.3$ & $28.3 \pm 2.1$ & $211.7 \pm 2.5$ & $-39.3 \pm 1.8$ & $40.6 \pm 3.2$ \\
\hline RPET-5 & $76.2 \pm 0.7$ & $184.3 \pm 0.6$ & $30.2 \pm 1.7$ & $211.1 \pm 0.8$ & $-41.8 \pm 1.4$ & $42.3 \pm 0.4$ \\
\hline
\end{tabular}


J.D. Badia, E. Strömberg, S. Karlsson, A. Ribes-Greus, The role of crystalline, mobile amorphous and rigid amorphous fractions in the performance of recycled poly (ethylene terephthalate) (PET). Polymer Degradation and Stability,97 (1) 98-107, 2012

In order to characterize the crystalline fraction of PET and its further recyclates, an approach by applying the Hoffman-Lauritzen nucleation theory [50-52] to relate the evolution of the lamellar thickness $\left(l_{C}\right)$ distribution with the reprocessing cycle was performed. The Thomson-Gibbs equation (Eq. 4) was used to assess the evolution of the lamellar thickness distribution:

$$
l_{C}(T m)=\left[\left(1-\frac{T_{m}}{T_{m}{ }^{0}}\right) \cdot \frac{\Delta h_{m V}}{2 \cdot \sigma_{e}}\right]^{-1}
$$

, where $T_{m}$ is the melting temperature; $T_{m}{ }^{0}$ is the equilibrium melting temperature of an infinite crystal (564 $\mathrm{K}) ; \sigma_{e}$ is the surface free energy of the basal plane where the chains fold $\left(0.106 \mathrm{~J} \cdot \mathrm{m}^{-2}\right) ; \Delta h_{m V}$ is the melting enthalpy per volume unit $\left(2.1 \cdot 10^{8} \mathrm{~J} \cdot \mathrm{m}^{-3}\right)$, both values obtained from studies by $\mathrm{Lu}$ and Hay [53]. Figure 8 represents the $l_{C}$ distribution for virgin and reprocessed PETs. In this figure, a prominent distribution related to the most probable lamellar size can be seen. A bimodal melting behaviour is displayed, which origin is still on debate [54-60]. Under isothermal DSC crystallization conditions, multiple melting behaviour of PET can be associated to a superposed melting-crystallization-remelting process [54-56], to the enthalpy recovery connected to the mobilization of the constrained $R A F$ [57] and/or the melting of two distinct crystal populations [58-60]. In any case, the effects of degradation could be monitored by the analysis of the melting endotherm at lower temperatures.

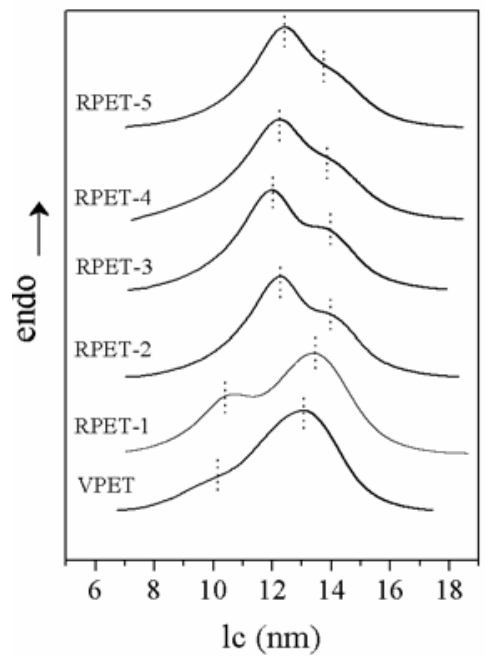

Figure 8. Lamellar thickness distributions of virgin and reprocessed PETs.

In order to better explain the effect of the degradation process on the crystalline fraction, a deconvolution procedure [61] was applied to the melting thermograms with the aim of individually characterizing the evolution of each $i$ area and its contribution to the overall effect using a partial areas study, as early proposed [8]. Fitting DSC procedures were performed by means of the software OriginLab OriginPro 8.0, which uses the Levenberg-Marquardt algorithm [62-63] to adjust the parameter of the fitting values in the iterative procedure. Areas were labelled as I and II from the lowest to the highest peak temperature $\left(T_{m}{ }^{i}\right)$, which are registered at Table 2, along with their melting onset temperatures $\left(T_{m 0}{ }^{i}\right)$, melting range temperatures $\left(\Delta T_{m}{ }^{i}\right)$ and average lamellar thicknesses $\left(l_{C}{ }^{i}\right)$. As well, the so-called relative partial crystallinity of each area [8] 
J.D. Badia, E. Strömberg, S. Karlsson, A. Ribes-Greus, The role of crystalline, mobile amorphous and rigid amorphous fractions in the performance of recycled poly (ethylene terephthalate) (PET). Polymer Degradation and Stability,97 (1) 98-107, 2012

was calculated by means of $X^{i}=X_{C}{ }^{i} / X_{C}=\Delta h_{m}{ }^{i} / \Delta h_{m}$, where $\Delta h_{m}{ }^{i}$ is the partial melting enthalpy of each area $i$, and compared to the full crystallinity degree $\left(X_{C}\right)$ in Figure 9.

Table 2. Melting calorimetric parameters after deconvolution

\begin{tabular}{|l|l|l|l|l|l|l|l|l|}
\hline Material & $\mathrm{T}_{\mathrm{m} 0}{ }^{\mathrm{I}}\left({ }^{\circ} \mathrm{C}\right)$ & $\left.\Delta \mathrm{T}_{\mathrm{m}}{ }^{\mathrm{I}}{ }^{\circ} \mathrm{C}\right)$ & $\mathrm{T}_{\mathrm{m}}{ }^{\mathrm{I}}\left({ }^{\circ} \mathrm{C}\right)$ & $\mathrm{l}_{\mathrm{C}}^{\mathrm{I}}(\AA)$ & $\mathrm{T}_{\mathrm{m} 0}{ }^{\mathrm{II}}\left({ }^{\circ} \mathrm{C}\right)$ & $\Delta \mathrm{T}_{\mathrm{m}}{ }^{\mathrm{II}}\left({ }^{\circ} \mathrm{C}\right)$ & $\mathrm{T}_{\mathrm{m}}{ }^{\mathrm{II}}\left({ }^{\circ} \mathrm{C}\right)$ & $\mathrm{l}_{\mathrm{C}}{ }^{\mathrm{II}}(\AA)$ \\
\hline VPET & $207.7 \pm 1.7$ & $39.1 \pm 1.4$ & $233.2 \pm 0.6$ & $9.8 \pm 0.12$ & $212.3 \pm 1.8$ & $47.4 \pm 2.1$ & $249.8 \pm 0.3$ & $13.8 \pm 0.13$ \\
\hline RPET-1 & $207.6 \pm 1.6$ & $41.7 \pm 2.5$ & $238.4 \pm 0.1$ & $10.8 \pm 0.02$ & $220.6 \pm 1.4$ & $39.4 \pm 1.8$ & $250.2 \pm 0.1$ & $14.0 \pm 0.05$ \\
\hline RPET-2 & $210.6 \pm 0.1$ & $41.8 \pm 2.2$ & $241.7 \pm 0.1$ & $11.6 \pm 0.01$ & $228.1 \pm 1.3$ & $35.4 \pm 0.2$ & $248.8 \pm 0.1$ & $13.5 \pm 0.01$ \\
\hline RPET-3 & $214.8 \pm 0.7$ & $35.6 \pm 0.4$ & $245.1 \pm 0.5$ & $12.4 \pm 0.14$ & $230.4 \pm 1.1$ & $29.6 \pm 1.1$ & $250.3 \pm 0.2$ & $14.1 \pm 0.08$ \\
\hline RPET-4 & $216.8 \pm 2.5$ & $34.7 \pm 0.7$ & $245.4 \pm 0.2$ & $12.6 \pm 0.06$ & $229.2 \pm 2.0$ & $30.8 \pm 2.8$ & $250.5 \pm 1.1$ & $14.1 \pm 0.31$ \\
\hline RPET-5 & $215.3 \pm 0.1$ & $35.0 \pm 0.3$ & $245.9 \pm 0.1$ & $12.7 \pm 0.02$ & $231.3 \pm 0.1$ & $29.4 \pm 0.6$ & $249.7 \pm 0.15$ & $13.8 \pm 0.04$ \\
\hline
\end{tabular}

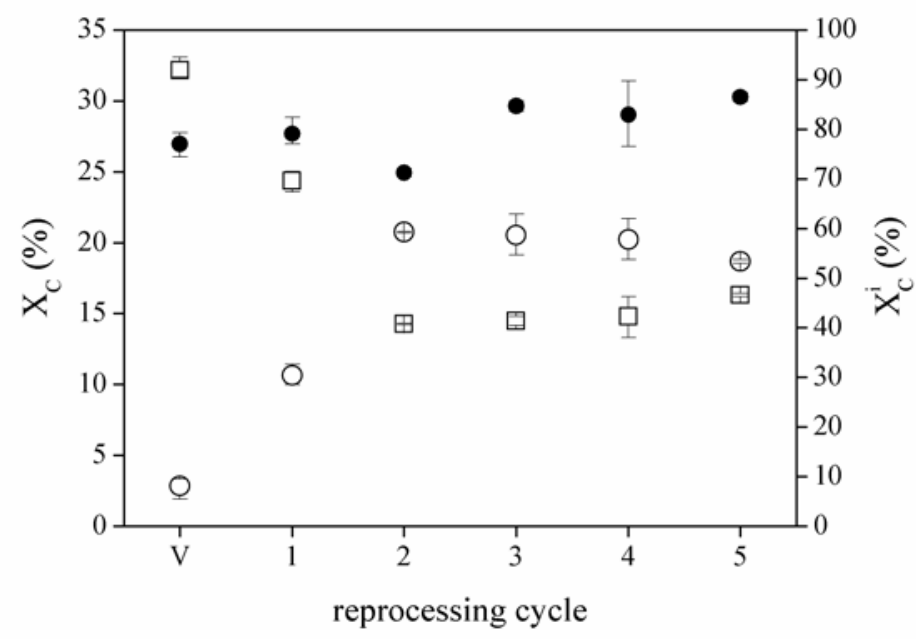

Figure 9. Evolution of relative partial crystallinity degrees

Despite $X_{C}$ was not sensitive to changes induced by reprocessing, the use of $X^{i}$ provided new insights on the formation of new crystals. The plots showed a continuous development of crystalline zones with lower lamellar size, especially from the first to the second recyclate. In connection with the results from the cooling DSC scan, the formation of shorter chains due to the scission of the backbone might act as nuclei upon crystallization, which promoted the growth of more crystalline domains. Both melting processes were also sharpened during reprocessing, with an overall $\Delta T_{m}{ }^{i}$ change of $\sim 5^{\circ} \mathrm{C}$ for area I, and $\sim 17^{\circ} \mathrm{C}$ for area II. As well, the onsets of melting of areas I and II were displaced $\sim 8{ }^{\circ} \mathrm{C}$ and $\sim 20^{\circ} \mathrm{C}$ to higher temperatures, respectively. Contrarily, the peak temperatures and their corresponding lamellar thicknesses did not follow 
J.D. Badia, E. Strömberg, S. Karlsson, A. Ribes-Greus, The role of crystalline, mobile amorphous and rigid amorphous fractions in the performance of recycled poly (ethylene terephthalate) (PET). Polymer Degradation and Stability,97 (1) 98-107, 2012

the same trend for both areas. Whereas $T_{m}{ }^{I I}$ remained around $250{ }^{\circ} \mathrm{C}$ for an average lamellar thickness of 13-14 nm, area I underwent an important increase of $\sim 8^{\circ} \mathrm{C}$ in its $T_{m}{ }^{I}$, from 207 to $215{ }^{\circ} \mathrm{C}$, related to the increase in $l_{C}^{I}$ from $9.8 \mathrm{~nm}$ in VPET to $12.7 \mathrm{~nm}$ in RPET-5. Considering the variation of $X^{I}$ respect to $X^{I I}$, the distribution balance of areas I/II (\%), which was 10/90 for VPET, decayed down to 60/40 after the second reprocessing cycle, and then smoothly decreased until a $~ 55 / 45$ for RPET-5. The rearrangement of new crystalline domains after each reprocessing cycle was promoted towards crystalline domains with smaller average sizes, being the tendency changed from RPET-1 to RPET-2. These variations in crystallinity, along with the relative variation between mobile and rigid amorphous fractions might role the performance of PET recyclates in applications at sub- $T_{g}$ temperatures.

\subsection{Segmental dynamics throughout the glass-rubber relaxation.}

The influence of multiple processing on the viscoelastic response of PET and its successive recyclates throughout the glass transition relaxation was evaluated by means of multi-frequency DMTA tests. The loss $\left(E^{\prime \prime}\right)$ moduli were recorded for PET reprocessed samples and their evolution as a function of temperature are displayed in Figure 10, respectively, for the analysis performed at a frequency of $1 \mathrm{~Hz}$. Results at the rest of applied frequencies showed similar tendencies.

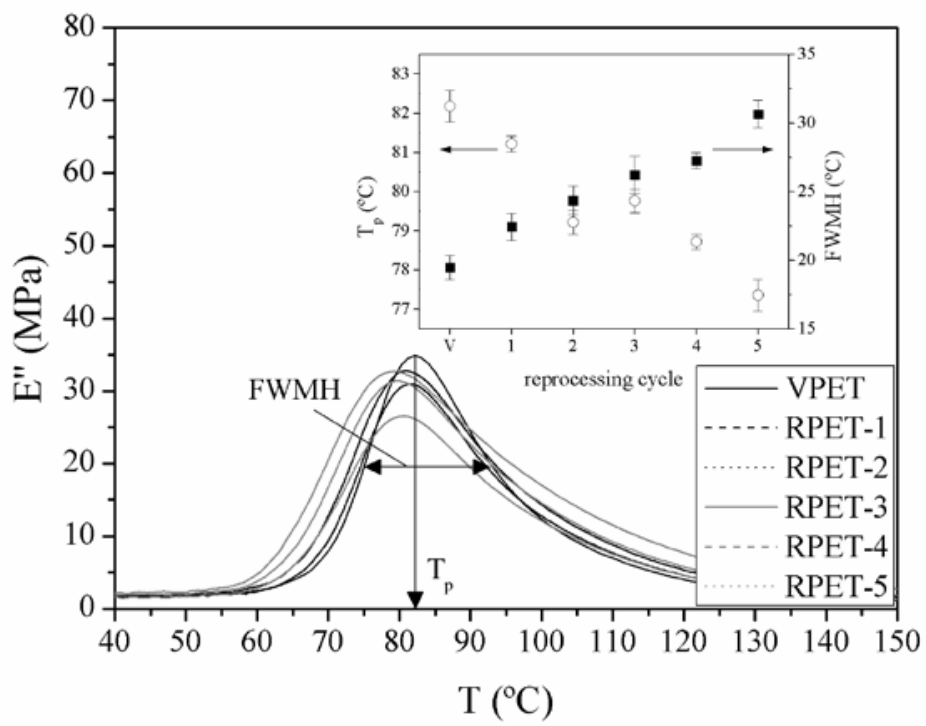

Figure 10. Evolution of loss moduli. Inset: variation of the peak temperatures and the full widths at medium height.

A prominent viscoelastic relaxation was displayed by virgin and reprocessed PETs, along a wide $\alpha$ relaxation that corresponds to the glass-rubber relaxation which extended from $60{ }^{\circ} \mathrm{C}$ to near $140{ }^{\circ} \mathrm{C}$. This relaxation is associated to the long-range segmental motions of the amorphous regions, which are dependent on the interaction with the rigid domains, comprised by both $R A F$ and crystalline fractions. In connection with FT-IR results, it was shown by NMR studies that the molecular motions of the $\alpha$-relaxations are driven by the trans/gauche isomerism of the ethylene groups of the glycol units of PET backbone [64]. The reprocessed samples generally presented a broader $E$ '” peak, as shown by the evolution of parameter that defines the Full Width at Medium High of $E^{\prime \prime}$ (labelled as FWMH in Fig 10). As well, the E', peak 
J.D. Badia, E. Strömberg, S. Karlsson, A. Ribes-Greus, The role of crystalline, mobile amorphous and rigid amorphous fractions in the performance of recycled poly (ethylene terephthalate) (PET). Polymer Degradation and Stability,97 (1) 98-107, 2012

temperature $T_{p}$ showed a displacement to lower values. These results are in agreement with other reports in literature [22, 65-66], which explained that the reduction of $T_{p}$ was indicative of dual amorphous fractions. The obtained decrease of $T_{p}$ thus also explained the increase of $R A F$ in the reprocessed samples.

The assessment of the glass-forming behaviour of PET provided more information regarding the influence of reprocessing to the mobility of PET chains throughout the glass-rubber relaxation. The strong-fragile glass former concept proposed by Angell [67] was followed, in which the variation of the viscosity with temperature could be explained under two border limits. A fragile glass-former experiences a dramatic loss of properties (rheological, mechanical...) throughout a specific short temperature interval, such as the glassrubber relaxation, while a strong glass-former maintains its properties without significant changes [68-69].

The temperature dependence of the relaxation times $\tau=\omega^{-1}=(2 \cdot \pi \cdot f)^{-1}$ of $E^{\prime \prime}$ spectra obtained during the glass transition relaxation at different angular/linear frequencies $\omega / f$ was fitted to the Vogel-FulcherTamman-Hesse [70-72] model. The so-called fragility index $m$ permitted an assessment of the deviation of $\tau(T)$ from the Arrhenius behaviour of polymers. It is defined as the characteristic slope of the fragility plot $\log \tau$ vs $T_{g} \cdot T^{1}$ and varies between two limiting values of 16 and $\geq 200$ for strong and fragile glass-formers, respectively [73-74]:

$$
m=\left.\frac{d \log (\tau)}{d(T g / T)}\right|_{T=T g}=\frac{B \cdot T}{\ln (10) \cdot\left(T g-T_{V F T H}\right)^{2}}(5)
$$

, where $B(\mathrm{~K})$ and $\mathrm{T}_{\mathrm{VFTH}}(\mathrm{K})$ are positive parameters specific for the material. Qualitatively, $B$ can be related to the topology of the theoretical potential energy surface of the system, where fragile systems present high density of energy minima, contrarily to strong systems which present lower density. Consequently, $m$ can be associated to the average height of the energy barrier that conformers may overcome to reach their energy minima. The evolution of $B$ and $T_{V F T H}$ is gathered in Table 3. Linear correlation values $\left(R^{2}\right)$ around 0.99 were obtained by the fitting to the VFTH model. In addition, the Angell's diagram is shown at Figure 11. The fragility index, obtained from the slope of the curves at $T=T_{g}$, decreased with each reprocessing cycle, as also given in Table 3. The influence of the chemistry of the backbone and of the side-groups on the fragility of polymers was thoroughly discussed by Kunal et al [75], stating that the fragility depended on the relative size balance between backbone and side groups. PET, due to its characteristic rigid backbone involving aromatic rings, is inherently fragile, associated to inefficient packing of rigid chains. In polymers with rigid backbones, studies in literature indicate that an increase in chain length lead to an increase in $m$ [76-77]. In this study, the decrease of $m$ was therefore indicative of cutbacks in the amorphous region MAF.

Table 3 also shows the results of the free volume coefficient $\phi$ obtained by Eq (6) and the parameter related to the apparent activation energy associated to the glass-rubber relaxation $E a_{G T}$ obtained after applying Eq (7).

$$
\phi=\frac{\left(T-T_{V F T H}\right)}{B}(6)
$$




$$
E a_{G T}=R \cdot \frac{d l n \tau}{d(1 / T)}=\frac{R \cdot B}{\left(1-\frac{T_{V F T H}}{T}\right)^{2}}
$$

Table 3. Results from the assessment of the segmental dynamics

\begin{tabular}{|c|c|c|c|c|c|c|c|}
\cline { 2 - 8 } \multicolumn{1}{c|}{} & \multicolumn{4}{c|}{ VFTH fitting } & \multicolumn{4}{c|}{ Calculated parameters } \\
\hline Material & $\mathrm{T}_{\mathrm{g}}\left({ }^{\circ} \mathrm{C}\right) *$ & $\mathrm{~T}_{\mathrm{VFTH}}\left({ }^{\circ} \mathrm{C}\right)$ & $\mathrm{R}^{2}$ & $\mathrm{~B}(\mathrm{~K})$ & $\Phi(\%)$ & Ea $\left(\mathrm{kJ} \cdot \mathrm{mol}^{-1}\right)$ & $\mathrm{m}$ \\
\hline VPET & $77.6 \pm 2.1$ & $42.0 \pm 1.7$ & 0.998 & 905.5 & 3.92 & 732 & 109 \\
\hline RPET-1 & $77.1 \pm 1.1$ & $41.5 \pm 1.2$ & 0.996 & 813.1 & 4.37 & 653 & 97 \\
\hline RPET-2 & $76.4 \pm 0.8$ & $39.9 \pm 0.5$ & 0.988 & 798.3 & 4.56 & 609 & 91 \\
\hline RPET-3 & $76.2 \pm 0.8$ & $39.9 \pm 0.3$ & 0.998 & 789.4 & 4.58 & 610 & 91 \\
\hline RPET-4 & $76.3 \pm 0.6$ & $38.9 \pm 0.6$ & 0.994 & 762.5 & 4.89 & 555 & 83 \\
\hline RPET-5 & $76.2 \pm 0.8$ & $37.9 \pm 0.1$ & 0.993 & 765.7 & 4.99 & 531 & 79 \\
\hline * Tg=Tp taken at the experiment at the lowest tested frequency: $0.01 \mathrm{~Hz}$ & & \\
\hline
\end{tabular}

The free volume is usually ascribed to the packing defects in the structure due to inhibition of the segmental mobility below $T_{g}$, and thus, due to reprocessing, the rearrangement of the chains may provoke molecularsize cavities. A slight increase of free volume was obtained, due to chain scission processes. This effect might promote the liability of gases to permeate through the polymer in packaging applications [25], therefore reducing the second-life performance of PET for similar purposes. On the other hand, the high apparent activation energies $\left(E a_{G T}\right)$ found for virgin PET, as observed for other studies [78-79], progressively decreased for each PET recyclate, since less energy would be required to control the movement of shorter chains with each reprocessing cycle. 


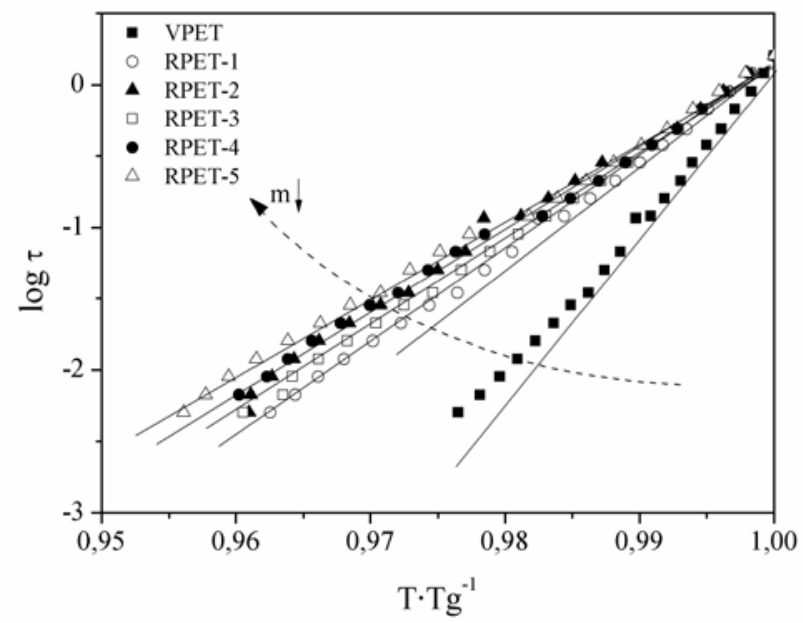

Figure 11. Angell's plot. Evolution of the fragility index m.

\subsection{The role of MAF and RAF on the mechanical performance PET}

The influence of the microstructural conformation of reprocessed PET was finally assessed on the mechanical performance by tensile and impact testing, and the study of the storage modulus ( $E$ ') spectra by DMTA.

Figure 12 shows the results obtained by traditionally used tensile and impact tests. On the one hand, a slight overall variation in the Young modulus ( $14 \%$ ) was registered for RPET-3,4,5 in comparison to that of VPET. On the other hand, the stress and elongation at break were clearly influenced by the reprocessing cycles, showing a critical drop from RPET-1 to RPET-2. The chemical and morphological changes induced by reprocessing gave out more brittle materials with lower ductility, as drawn from the variation of the impact strength value, which was halved for the second recyclate. These results were in agreement with those given by other authors for PET [12], PS [5] or PE [4] who did not observed significant changes in the elastic modulus due to reprocessing, though the elongation at break strongly diminished. The thermomechanical degradation effects on the macroscopic mechanical properties could be explained by the role of the increasing $R A F$ throughout the processing cycles. A strain-induced crystallization may had occurred upon tensile testing below $T_{g}$, where part of the newly formed $R A F$ due to reprocessing may enhance interactions and entanglements between chains, as well as act as precursor of new nucleation sites, enhancing the growth of the crystalline phase during tensile testing, as supported by X-ray Diffraction studies [80-82]. Furthermore, Schick et al suggested that the RAF itself may vitrify [21], thus increasing the rigidity of PET upon drawing, acting as topological constraints against deformation transforming the initial ductile behaviour of PET, breaking the probe by means of a brittle fracture with lower applied stress. 

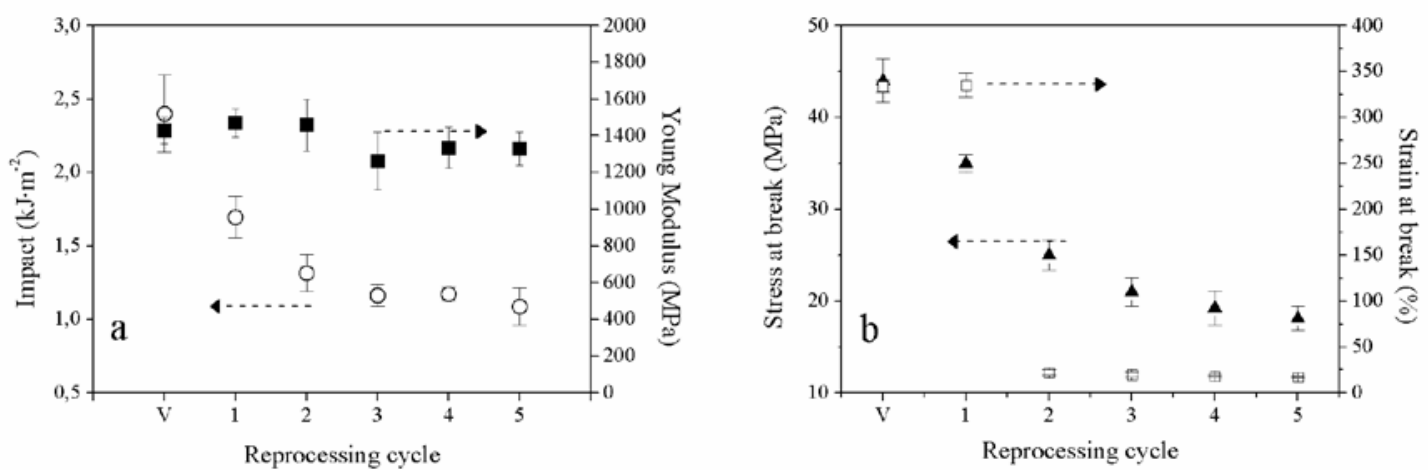

Figure 12. Variation of mechanical properties as analyzed by tensile and impact testing.

Concerning the role of $M A F$, its diminution was more significant upon glass-rubber relaxation. Figure 13 represents the viscoelastic storage modulus (E') of virgin PET and its five successive recyclates obtained by DMTA performed at $1 \mathrm{~Hz}$. Experiments performed at the rest of frequencies showed similar behaviours. The mechanical strength $\Delta E^{\prime}$, calculated as the difference between the $E^{\prime}$ values at 40 and $170{ }^{\circ} \mathrm{C}$, was evaluated. Taking into account that the crystalline fraction remained scarcely changed along the reprocessed materials, the role of $M A F$ drove the increase of $\triangle E^{\prime}$. In connection with DSC results, the significant loss of MAF negatively affected the elastic behaviour of PET, since less non-constrained amorphous chains were subjected to the tension of the experiment, therefore increasing $E$ ' and subsequently $\Delta E^{\prime}$ ' showed an overall increase of 20 \% from VPET to RPET-5.

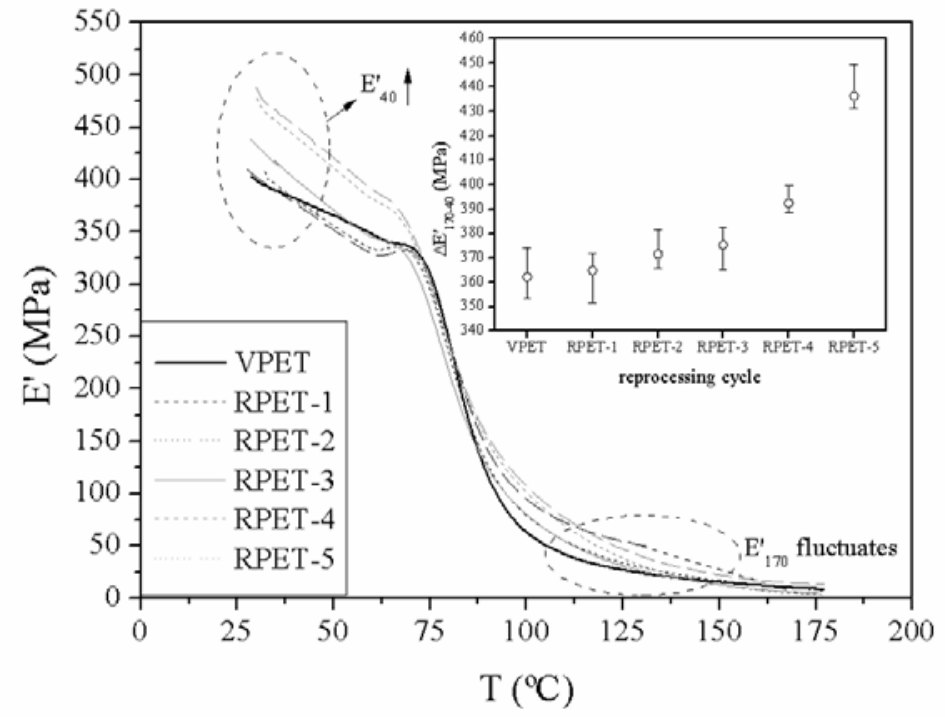

Figure 13. Evolution of storage moduli at $1 \mathrm{~Hz}$. Inset: variation of mechanical stresses through the reprocessing cycles. 
J.D. Badia, E. Strömberg, S. Karlsson, A. Ribes-Greus, The role of crystalline, mobile amorphous and rigid amorphous fractions in the performance of recycled poly (ethylene terephthalate) (PET). Polymer Degradation and Stability,97 (1) 98-107, 2012

\section{Conclusions}

Thermo-mechanical degradation induced by successive injection moulding cycles affected PET structure by mainly reducing initial diethylene glycol domains to ethylene glycol units in the flexible part of the PET backbone. Besides, chain scission processes produced -OH terminated species with shorter chain length, which increased the yellowish aspect of PET recyclates and reduced its molar mass.

The effects of reprocessing on the microstructure were assessed taking into account a three-fraction model involving crystalline (C), mobile amorphous fraction (MAF) and rigid amorphous fraction (RAF). Degradation was mainly driven by the cleavage of MAF and the tendency of shortened PET chains to reorganize into $R A F$. As well, the crystalline fraction was formed earlier and steeper after each reprocessing step. Despite the overall crystallinity fraction scarcely changed, a deep characterization of the bimodal melting behaviour indicated the arrangement of crystalline domains with smaller lamellar thickness.

The rearrangement after reprocessing affected to the viscoelastic and mechanical performance. The reduction of the MAF due to chain scissions increased the free volume of the new recyclates, which relaxations from the glassy to a rubbery state were overcome easier and involving less activation energies, due to a decrease in dynamic fragility. The role of $R A F$ was relevant in the behaviour at break during macroscopic mechanical testing, due to it might act as precursor of crystallinity and/or vitrify, thus promoting a strain-induced crystallization during tensile drawing and subsequently enhancing the embrittlement of PET.

The microstructural study of the balance between C, MAF and RAF permitted to establish suitable degradation parameters for the control of the quality of PET recyclates. The study of all properties showed a significant loss after the application of the first reprocessing step, thus showing a sort of threshold for PET to be recovered by further mechanical recycling. 
J.D. Badia, E. Strömberg, S. Karlsson, A. Ribes-Greus, The role of crystalline, mobile amorphous and rigid amorphous fractions in the performance of recycled poly (ethylene terephthalate) (PET). Polymer Degradation and Stability,97 (1) 98-107, 2012

\section{Acknowledgements}

The authors would like to acknowledge the Spanish Ministry of Science and Innovation for the financial support through the Research Project UPOVCE-3E-013 and for the funding of a predoctoral research position to J.D. Badía by means of the FPU program conceded by the Spanish Ministry for Education. Catalana de Polimers, S.A. and AIMPLAS are acknowledged for providing and processing the material, respectively. Mr. Peter Kaali is thanked for carrying out impact tests and Mrs. Sevil Atari Jabarzadeh for helping with SEM analysis. The authors thank the financial support of the Generalitat Valenciana through the ACOMP/2011/189 and the Gerónimo Forteza grant to J.D. Badía. Universitat Politècnica de València (UPV, Spain) is thanked for additional support through the PAID 05-09-4331 program and Royal Institute of Technology (KTH, Sweden) is thanked for additional economical support. 
J.D. Badia, E. Strömberg, S. Karlsson, A. Ribes-Greus, The role of crystalline, mobile amorphous and rigid amorphous fractions in the performance of recycled poly (ethylene terephthalate) (PET). Polymer Degradation and Stability,97 (1) 98-107, 2012

\section{REFERENCES}

1. D.M. Fann, S. K. Huang, J.Y. Lee. Kinetics and thermal crystallinity of recycled PET. II. Topographic study on thermal crystallinity of injection molded recycled PET. Journal of Applied Polymer Science 1996; 61; 261-271

2. R. J. Ehrig. Plastics Recycling: Products and Processes. New York : Hanser Publishers, 1992.

3. P. P. Klemchuck. Polymer Stabilization and Degradation. Washington : ACS, 1985.

4. E. Strömberg, S. Karlsson. The design of a test protocol to model the degradation of polyolefins during recycling and service life. Journal of Applied Polymer Science 2099; 112: 1835-1844.

5. F. Vilaplana, A. Ribes-Greus, S. Karlsson. Degradation of recycled high-impact polyestyrene .Simulation by reprocessing and thermo-oxidation. Polymer Degradation and Stability 2006; 91: 21632170.

6. F. Vilaplana, S. Karlsson. A. Ribes-Greus. Changes in the micro-structure and morphology of highimpact polystyrene subjected to multiple processing and thermo-oxidative degradation. European Polymer Journal 2007; 43: 4371-4381.

7. N. Yarahmadi, I. Jakubowicz, T. Gevert. Effects of repeated extrusion on the properties and durability of rigid PVC scrap. Polymer Degradation and Stability 2001; 73: 93-99.

8. J.D. Badía, F. Vilaplana, S. Karlsson, A. Ribes-Greus. Thermal analysis as a quality tool for assessing the influence of thermo-mechanical degradation on recycled poly(ethylene terephthalate). Polymer Testing 2009; 28: 169-175.

9. J.D. Badía, E. Strömberg, A. Ribes-Greus, S. Karlsson. A statistical design of experiments for optimizing the MALDI-TOF-MS sample preparation of polymers. An application in the assessment of the thermo-mechanical degradation mechanisms of poly (ethylene terephthalate). Analytica Chimica Acta 2011; 692: 85-95

10. S.D.Mancini, M. Zanin. Consecutive Steps of PET Recycling by Injection: Evaluation of the Procedure and of the Mechanical Properties. Journal of Applied Polymer Science 2000; 76: 266-275.

11. F. La Mantia, M. Vinci. Recycling poly(ethylene terephthalate). Polymer Degradation and Stability 1994; 45: 121-125.

12. N. Torres, J.J. Robin, B. Boutevin. Study of thermal and mechanical properties of virgin and recycled poly(ethylene terephthalate) before and after injection molding. European Polymer Journal 2000; 36: 2075-2080.

13. W. Romao, M. F. Franco, Y. E. Corilo, M.N. Eberlin, M.A.S, Spinacé, M.A. De Paoli. Poly(ethylene terephthalate) thermo-mechanical and thermo-oxidative degradation mechanisms. Polymer Degradation and Stability 2009; 94: 1849-1859.

14. A. Oromiehie, A. Mamizadeh. Recycling PET beverage bottles and improving properties. Polymer International 2004; 53: 728-732.

15. A. Pawlak, M. Pluta, J. Morawiec, A. Galeski, M. Pracella. Characterization of scrap poly(ethylene terephthalate). European Polymer Journal 2000; 36: 1875-1884.

16. F. Awaja, D. Pavel. Recycling of PET. European Polymer Journal 2005; 41: 1453-1477.

17. Y. Fu, B. Annis, A. Boller, Y. Jin, B. Wunderlich. Analysis of structure and properties of poly(ethylene terephthalate). Journal of Polymer Science: Polymer Physics 1994; 32: 2289-2306. 
J.D. Badia, E. Strömberg, S. Karlsson, A. Ribes-Greus, The role of crystalline, mobile amorphous and rigid amorphous fractions in the performance of recycled poly (ethylene terephthalate) (PET). Polymer Degradation and Stability,97 (1) 98-107, 2012

18. Y. fu, W. R. Busing, Y. Jin, K. A. Affholter, B. Wunderlich. Structure analysis of the non-crystalline material in poly(ethylene terephthalate) fibers. Macromolecular Chemistry Physics 1994; 195: 803-822.

19. A. Flores, M. Pieruccini, N. Stribeck, S.S. Funari, E. Bosch F.J. Baltá-Calleja. Structure formation in poly (ethylene terephthalate) upon annealing as revealed by microindentation hardness and X-ray scattering. Polymer 2005; 24: 9404-9410.

20. K.C. Cole, A. Aiji, E. Pellerin. New insights into the development of ordered structure in poly(ethylene terephthalate). Results from external reflection infrared spectroscopy. Macromolecules 2002; 32: 770-784.

21. C. Schick, A. Wurm, A. Mohamed. Vitrification of the rigid amorphous phase in semicrystalline polymers revealed from frequency-dependent heat capacity. Colloid Polymer Science 2001; 279: 800 806.

22. B. Wunderlich. Reversible crystallization and the rigid amorphous phase in semicrystalline polymers. Polymer Science 2003; 28: 383-450.

23. P. G. Karagiannidis, A. C. Stergiou, G. P. Karayannidis. Study of crystallinity and thermomechanical analysis of annealed poly(ethylene terephthalate) films. European Polymer Journal 2008; 44: 1475-1486.

24. R. Rastogi, W. P. Vellinga, S. Rastogi, C. Schick, H.E.H. Meijer. The three-phase structure and mechanical properties of poly(ethylene terephthalate). Journal of Polymer Science: Part B: Polymer Physics 2004; 42: 2092-2106.

25. J. Lin, S. Shenogin, S. Nazarenko. Oxygen solubility and specific volume of rigid amorphous fraction in semicrystalline poly(ethylene terephthalate). Polymer 2002; 43: 4733-4743.

26. Z. Xia, H.J. Sue, Z. Wang, C. A. Avila-Orta, B. S. Hsiao. Determination of crystalline lamellar thickness in poly(ethylene terephthalate) using small-angle x-ray scattering and transmission electron microscopy. Journal of Macromolecular Science, Part B: Physics 2001; 40: 625-638.

27. H. G. Haubruge, A.M. Jonas, R. Legras. Morphological Study of Melt-Crystallized Poly(ethylene terephthalate). A. Comparison of Transmission Electron Microscopy and Small-Angle X-ray Scattering of Bulk Samples. Macromolecules 2004; 37: 126-134.

28. A. Bartolotta, G. Di Marco, F. Farsaci, M. Lanza, M. Pieruccini. DSC and DMTA study of annealed cold-drawn PET: a three phase model interpretation. Polymer 2003; 44: 5771-5777.

29. B. B. Sauer, B.S. Hsiao, B S. Effect of the heterogeneous distribution of lamellar stacks on amorphous relaxations in semicrystalline polymers Polymer 1995; 36: 2553-2558.

30. ISO 291:1997. Plastics - standard atmospheres for conditioning and testing.

31. ISO 527-2: Plastics -- Determination of tensile properties -- Part 2: Test conditions for moulding and extrusion plastics. 1993.

32. ISO 179-1 Plastics -- Determination of Charpy impact properties -- Part 1: Non-instrumented impact test. 2010.

33. K. C. Cole, J. Guèvremont, A. Aiji, M.M. Dumoulin. Characterization of surface orientation in poly(ethylene terephthalate) by front-surface reflection infrared spectroscopy. Applied Spectroscopy 1994; 48: 1513-1521.

34. D.J. Walls. Application of ATR-IR to the analysis of surface structure and orientation in uniaxially drawn poly(ethyleneterephthalate). Applied Spectroscopy 1991; 45: 1193-1198 
J.D. Badia, E. Strömberg, S. Karlsson, A. Ribes-Greus, The role of crystalline, mobile amorphous and rigid amorphous fractions in the performance of recycled poly (ethylene terephthalate) (PET). Polymer Degradation and Stability,97 (1) 98-107, 2012

35. ISO 1628-1: 2009. Plastics -- Determination of the viscosity of polymers in dilute solution using capillary viscometers -- Part 1: General principles.

36. L H. Sperling. Introduction to physical polymer science. 4th. Hoboken : John Wiley \& Sons, 2006.

37. S. Fakirov. Polyethylene terephthalate. In: O Olabisi. Handbook of thermoplastics. New York : Marcel Dekker, 1994, p. 451.

38. S. A. Jabarin. Crystallization kinetics of polyethylene terephthalate, I. Isothermal crystallization from the melt. Journal of Applied Polymer Science 1987; 34: 85-96.

39. N. B. Sanches, M. L. Dias, E. B. Pacheco. Comparative techniques for molecular weight evaluation of poly(ethylene terephthalate) (PET). Polymer testing 2005; 24: 688-693.

40. ISO 11357-2 Plastics. Differential Scanning Calorimetry. Part 2- Determination of the glass transition. 1999.

41. B. Wunderlich. Macromolecular Physics. A PressNew York and London : 1973, Vol. 1, p. 389.

42. B. Wunderlich, The Athas Data Base on Heat Capacities of Polymers. Pure and Applied Chemistry 1995; 67, 1019-1026 . http://web.utk.edu/athas/databank/.

43. F.J. Boerio, S.K. Bahl, G.E. McGraw. Vibrational analysis of polyethylene terephthalate and its deuterated derivatives. Journal of Polymer Science, Polymer Physics 1976; 14: 1029-1046.

44. A. Miyake. The infrared spectrum of polyethylene terephthalate. I The effect of crystallization. Journal of Polymer Science 1959; 38: 479-495.

45. B. J. Holland, J. N. Hay. Analysis of comonomer content and cyclic oligomers of poly(ethylene terephthalate).Polymer 2002; 43: 1797-1804.

46. B. García-Gaitán, M. D. P. Pérez-González, A. Martínez-Richa, G. Luna-Bárcenas, S. M. NuñoDonlucas, Segmented block copolymers of poly(ethylene glycol) and poly(ethylene terephthalate). Journal of Polymer Science Part A: Polymer Chemistry 2004 ,42: 4448-4457.

47. C. F. Ciolacu, N. R. Choudhury, N. K. Dutta. Colour formation in poly(ethylene terephthalate) during melt processing. Polymer Degradation and Stability 2006; 91:. 875-885.

48. J. D. Menczel, M. Jaffe. How did we find the rigid amorphous phase? Journal of Thermal Analysis and Calorimetry 2007; 89: 357-362.

49. W. Romao, M. F. Franco, M. I. M.S. Bueno, M. A. De Paoli, Distinguishing between virgin and postconsumption bottle-grade poly (ethylene terephthalate) using thermal properties, Polymer Testing 2010; 29: 879-885-

50. J.L. Lauritzen and J.D. Hoffman. Theory of formation of polymer crystals with folded chains in dilute solution. Journal of Research of the National Bureau of Standards 1960; 64: 73-102.

51. J.L. Lauritzen and J.D. Hoffman. Crystallization of bulk polymers with chain folding: theory of growth of lamellar spherulites. Journal of Research of the National Bureau of Standards 1961; 65: 297336.

52. J.D. Hoffman, G.T. Davis, J. L. Lauritzen, The rate of crystallization of linear polymers with chain folding. Treatise on solid state chemistry. In: N.B. Hannay, Crystalline and noncrystalline solids vol. 3, Plenum Press, New York (1976). Hoffman. pp. 497-614. 
J.D. Badia, E. Strömberg, S. Karlsson, A. Ribes-Greus, The role of crystalline, mobile amorphous and rigid amorphous fractions in the performance of recycled poly (ethylene terephthalate) (PET). Polymer Degradation and Stability,97 (1) 98-107, 2012

53. X.F. Lu, J.N. Hay, Isothermal crystallization kinetics and melting behaviour of poly(ethylene terephthalate). Polymer 2001; 42; 9423-9431.

54. Z. G.Wang, B. S. Hsiao, B. B. Sauer, W.G. Kampert. The nature of secondary crystallization in poly(ethylene terephthalate). Polymer 1999; 40: 4615-4627.

55. A. A. Minakov, D. A. Mordvintsev, C. Schick. Melting and reorganization of poly(ethylene terephthalate) on fast heating (1000 K/s). Polymer 2004; 45: 3755-3763.

56. V. H. G. Zachmann, H. A. Stuart. Schmelz- und kristallisationserscheinungen bei makromolekularen substanzen. V. Partielles schmelzen und neukristallisieren von terylen. Die Makromolekulare Chemie 1960; 41: 148-173.

57. M. Song. Rigid amorphous phase and low temperature melting endotherm of poly(ethylene terephthalate) studied by modulated differential scanning calorimetry. Journal of Applied Polymer Science 2001; 81: 2779-2785.

58. G. E. Sweet, J. P. Bell. Multiple endotherm melting behavior in relation to polymer morphology. Journal of Polymer Science. Part A-2: Polymer physics 1969, 10: 1273-1283.

59. R. C. Roberts. Poly(ethylene terephthalate) II—Morphological changes on annealing. Polymer 1969; 10: 117-125.

60. S. Tan, A. Su, W. Li, E. Zhou. New insight into melting and crystallization behavior in semicrystalline poly (ethylene terephthalate). Journal of Polymer Science: Part B: Polymer physics 2000; 38: $53-60$

61. L. Santonja-Blasco, R. Moriana, J. D. Badía, A. Ribes-Greus. Thermal analysis applied to the characterization of degradation in soil of polylactide: I. Calorimetric and viscoelastic analyses. Polymer Degradation and Stability 2010; 95: 2185-2191.

62. K. Levenberg. A method for solution of certain non-linear problems in least squares. Quaterly of Applied Mathematics 1944: 2: 164-168.

63. D. W. Marquardt. An algorithm for the least-squares estimation of non-linear parameters. SIAM Journal of Applied Mathematics 1963; 11:. 431-441.

64. A. D. English. Macromolecular dynamics in solid poly(ethylene terephtahalate): ${ }^{1} \mathrm{H}$ and ${ }^{13} \mathrm{C}$ solidstate NMR. Macromolecules 1984; 17: 2182-2192.

65. J. Zhao, J. Wang, C. Li, Q. Fan. Study of the amorphous phase in semicrystalline poly(ethylene terephthalate) via physical aging. Macromolecules 2002; 35: 3097-3103.

66. J. Zhao, R. Song, Z. Zhang, X. Linghu, Z. Zheng, Q. Fan, A study of the physical aging in semicrystalline poly(ethylene terephthalate) via differential scanning Calorimetry. Macromolecules 2001; 34: 343-345.

67. C.A. Angell. Relaxation in liquids, polymers and plastic crystals - strong/fragile patterns and problems. Journal of Non-Crystalline Solids 1991; 13: 131-133.

68. M.E. Godard, J.M. Saiter. Fragility and non-linearity in polymethyl( $\alpha$-n-alkyl) acrylates. Journal of Non-Crystalline Solids 1998; 235: 635-639..

69. A. Saiter, M. Hess, N. A. D'Souza, J. M. Saiter. Entropy and fragility in vitreous polymers. Polymer 2002; 43: 7497

70. H. Vogel, The temperature dependence law of the viscosity of fluids. Phys Z 1921; 22: 645-646. 
J.D. Badia, E. Strömberg, S. Karlsson, A. Ribes-Greus, The role of crystalline, mobile amorphous and rigid amorphous fractions in the performance of recycled poly (ethylene terephthalate) (PET). Polymer Degradation and Stability,97 (1) 98-107, 2012

71. G.S. Fulcher. Analysis of recent measurements of the viscosity of glasses. Journal of the American Ceramic Society 1925; 8: 339-355.

72. G. Tammann and G. Hesse. The dependence of viscosity upon temperature of supercooled liquids. Z Anorg Allg Chem. 1926; 156: 245-257.

73. R. Bohmer, C.A. Angell in: R. Richert , A. Blumen, Disorder effects on relaxational processes, Springer, Berlin, 1994.

74. T.A. Vilgis. Strong and fragile glasses: A powerful classification and its consequences. Physical Review B 1993; 47 2882-2885.

75. K. Kunal, C. G. Robertson, S. Pawlus, S. F. Hahn, A. P. Sokolov. Role of Chemical Structure in Fragility of Polymers: A Qualitative Picture. Macromolecules 2008; 41: 7232-7238.

76. P.G. Santangelo, C. M. Roland. Molecular weight dependence of fragility in polystyrene. Macromolecules 1998; 31: 45814585.

77. M. L. Williams, R. F. Landel, J. D. Ferry. The temperature dependence of relaxation mechanisms in amorphous polymers and other glass-forming liquids. Journal of the American Chemistry Society 1955; 77: 3701-3707.

78. N. M. Alves, J. F. Mano, J. L. Gómez-Ribelles. Molecular mobility in polymers studied with thermally stimulated recovery. II. Study of the glass transition of a semicrystalline PET and comparison with DSC and DMA results. Polymer 2002; 43: 3627-3633.

79. M. Cristea, D. Ionita, B. C. Simionescu. A new insight in the dynamo-mechanical behavior of poly(ethylene terephthalate). European Polymer Journal 2010; 46: 2005-2012.

80. G. E. Welsh, D. J. Blundell, A. H. Windle. A Transient Liquid Crystalline Phase as a Precursor for the Crystallization in Random Co-polyesters Fibers. Macromolecules 1998; 31: 7562-7565.

81. A. Mahendrasingam, C. Martin, W. Fuller, D. J. Blundell, R. J. Oldman, D. H. MacKerron, J. L. Harvie, C. Riekel. Observation of a transient structure prior to strain-induced crystallization in poly(ethylene terephthalate). Polymer 2000; 41: 1217-1221.

82. S. Ran, Z. Whang, C. Burger, B. Chu, B. H. Hsiao. Mesophase as the precursor for Strain-Induced Crystallization in Amorphous Poly(Ethylene Terephthalate) Film. Macromolecules 2002; 35: 1010210107. 